DRAFT VERSION NOVEMBER 12, 2018

Preprint typeset using LTEX style emulateapj v. 11/10/09

\title{
GALEX J201337.6+092801: THE LOWEST GRAVITY SUBDWARF B PULSATOR
}

\author{
R. H. ØSTENSEN ${ }^{1}$, P. I. PÁPICS ${ }^{1}$, R. OReiro ${ }^{1,2}$, M. D. ReEd ${ }^{3}$, A. C. QUINT ${ }^{3}$, J. T. GILKer ${ }^{3}$, L. L. HiCKS ${ }^{3}$, \\ A. S. BARAN ${ }^{4,5}$, L. Fox MAChADO ${ }^{6}$, T. A. OtTOSEN ${ }^{7,8}$ And J. H. Telting ${ }^{7}$ \\ ${ }^{1}$ Instituut voor Sterenkunde, K.U. Leuven, Celestijnenlaan 200D, 3001 Leuven, Belgium; roy@ster.kuleuven.be \\ ${ }^{2}$ Instituto de Astrofísica de Andalucía, Glorieta de la Astronomía s/n, 18008 Granada, Spain \\ ${ }^{3}$ Department of Physics, Astronomy, and Materials Science, Missouri State University, Springfield, MO 65804, USA \\ ${ }^{4}$ Mt. Suhora Observatory, Cracow Pedagogical University, Podchorazych 2, 30-084 Krakow, Poland \\ ${ }^{5}$ Department of Physics and Astronomy, Iowa State University, Ames, IA 50011, USA \\ ${ }^{6}$ Observatorio Astronómico Nacional, Universidad Nacional Autónoma de México, Ensenada, BC 22860, Mexico \\ ${ }^{7}$ Nordic Optical Telescope, 38700 Santa Cruz de La Palma, Spain and \\ ${ }^{8}$ Department of Physics and Astronomy, Aarhus University, 8000 Aarhus C, Denmark \\ Draft version November 12, 2018
}

\begin{abstract}
We present the recent discovery of a new subdwarf B variable (sdBV), with an exceptionally low surface gravity. Our spectroscopy places it at $T_{\text {eff }}=32100 \pm 1000, \log g=5.15 \pm 0.20$, and $\log \left(N_{\mathrm{He}} / N_{\mathrm{H}}\right)=-2.8 \pm 0.2$. With a magnitude of $B=12.0$, it is the second brightest V361 Hya star ever found. Photometry from three different observatories reveals a temporal spectrum with eleven clearly detected periods in the range 376 to $566 \mathrm{~s}$, and at least five more close to our detection limit. These periods are unusually long for the V361 Hya class of short-period sdBV pulsators, but not unreasonable for $p$ - and $g$-modes close to the radial fundamental, given its low surface gravity. Of the $\sim 50$ short-period sdB pulsators known to date, only a single one has been found to have comparable spectroscopic parameters to GALEX J201337.6+092801(J20136+0928, for short). This is the enigmatic high-amplitude pulsator V338 Ser, and we conclude that J20136+0928 is the second example of this rare subclass of sdB pulsators located well above the canonical extreme horizontal branch in the HR diagram.
\end{abstract}

Subject headings: subdwarfs - stars: early-type - stars: variables: general - stars: individual: GALEX J201337.6+09280

\section{INTRODUCTION}

The subdwarf B (sdB) stars are generally known to be core-helium-burning stars with inert hydrogen-dominated envelopes of very low mass. This composition places them on the hot extension of the horizontal branch, the extreme horizontal branch (EHB; Heber 1986, 2009). Their low-mass envelopes prevent them from reaching a second giant stage, and instead, after their core helium is exhausted, they expand only briefly, never exceeding more than about a third of a solar radius, before contracting and passing on towards higher effective temperatures and gravities. Thus, an sdB star evolves into the hotter sdO population before reaching degeneracy and the associated white dwarf (WD) cooling track (Dorman et al. 1993).

While the future evolution of $\mathrm{sdB}$ stars is unproblematic, exactly how they lose their envelopes during their first red giant (RGB) stage is more complicated. If the star has a close low-mass companion, mass transfer on the tip of the RGB will quickly pull it into the envelope. As friction transfers angular momentum from the orbit to the envelope, it will spin up and be ejected from the system. If the core has become massive enough for helium to ignite $\left(\sim 0.47 \mathrm{M}_{\odot}\right)$, the result will be a very close $\mathrm{sdB}+\mathrm{dM}$ system. These binaries have spectacular reflection effects, and a total of 14 such objects are described in the literature; 13 are listed in For et al. (2010) and the most recent one in Østensen et al. (2010a). While such $\mathrm{sdB}+\mathrm{dM}$ binaries make out only a tiny fraction of the total sdB population, cases where the companion responsible for ejecting the envelope is a low-mass WD, appear to be much more common. Maxted et al. (2001) found that 21 of 36 stars in their radial velocity study are in short period systems, most likely with WD companions.

If the companion is more massive than the subdwarf (at least at the end of mass transfer), the orbit will have expanded to more than a hundred days. Such orbits are hard to measure, but the companion is easily detectable spectroscopically or from infrared excess. Napiwotzki et al. (2004) found that more than a third of their sdB sample show the spectroscopic signature of main sequence companions, while Reed \& Stiening (2004), using 2MASS photometry, inferred that about half of the sdBs in the field are likely to be of this type. Thus, the vast majority of sdB stars in the field must be in binary systems. However, a few pulsating sdBs have been studied in such detail that any companion close enough to have interacted during the RGB stage can be ruled out. A possible explanation for such single sdB stars can be the merger of two low-mass WDs. These three scenarios are all investigated in detail in the binary population study of Han et al. (2002, 2003). They find that while the first two scenarios must have sdBs with masses close to the He-flash mass of $0.47 \mathrm{M}_{\odot}$, the merger scenario can produce sdBs with a broad distribution of masses ranging from 0.4 to $0.7 \mathrm{M}_{\odot}$.

While most sdB stars form a band in the $T_{\text {eff }}-\log g$ diagram that is consistent with a mass close to the canonical $0.47 \mathrm{M}_{\odot}$, and various envelope thicknesses up to the H-burning limit, a few exceptions are found. One such is V338 Ser, discovered to be a high amplitude sdB pulsator by Koen et al. (1998). A multi-site campaign devoted to this pulsator (Kilkenny et al. 1999) found more than fifty pulsation frequencies in the range $365-529 \mathrm{~s}$, which now defines the long-period edge for the V361 Hya group of short-period sdB variables (sdBVs). Time-resolved spectroscopy of V338 Ser was analysed by Tillich et al. (2007), and most recently Østensen et al. (2010c) 


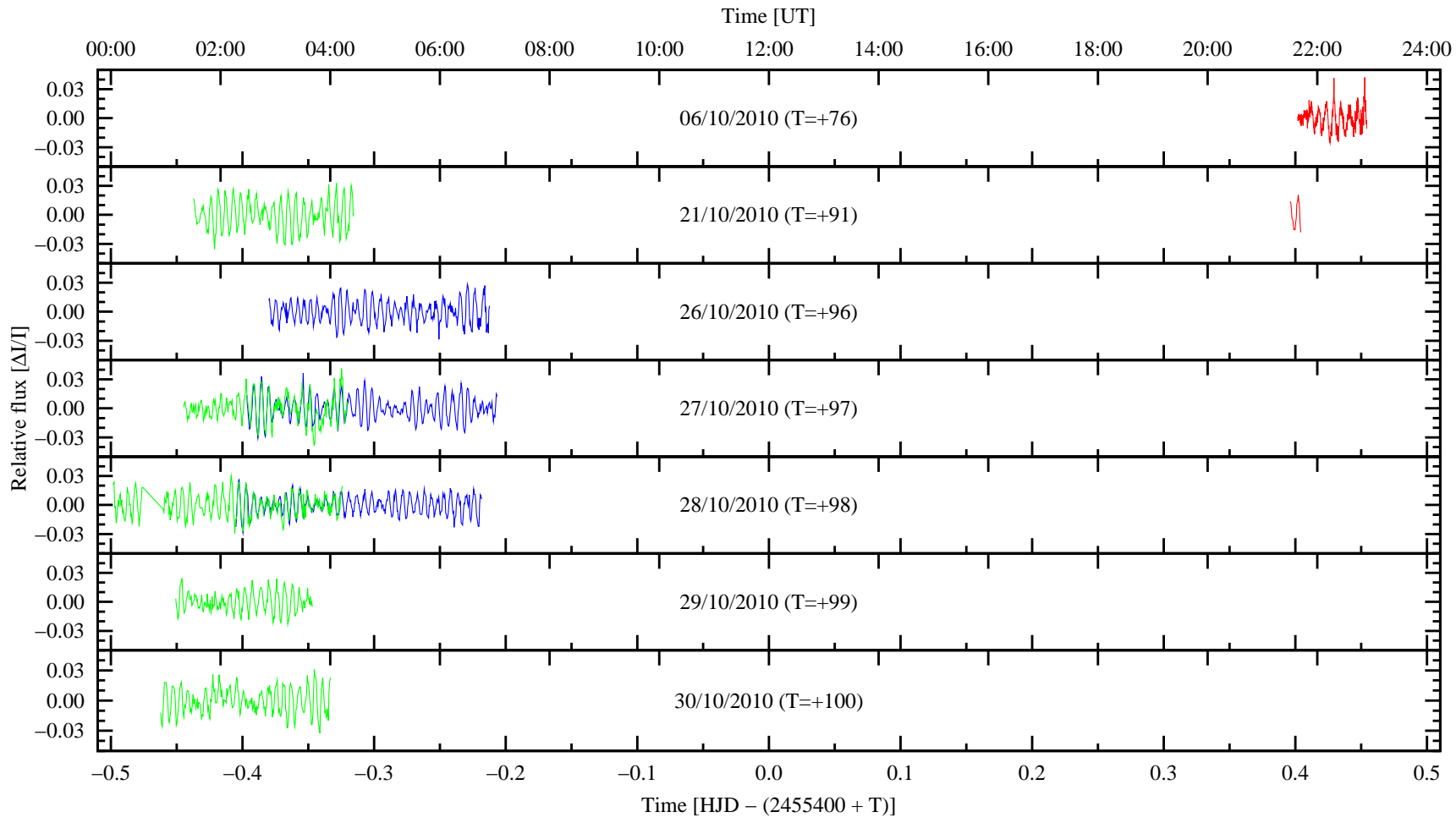

FIG. 1.- Light curves of J20136+0928. The top panel represents the discovery light curve. In the online version the different data sets are shown in different colors (Mercator; red, Baker; green, SPM; blue).

performed high-resolution time-resolved spectroscopy. Its unusually low gravity was discussed briefly by Østensen (2009), proposing that it may be a core-He-burning EHB star with a mass larger than the canonical, rather than a shell-Heburning post-EHB star as suggested by Koen et al. (1998). Asteroseismic solutions supporting this hypothesis were recently presented by van Grootel et al. (2010), implying a mass of $0.76 \mathrm{M}_{\odot}$, and an envelope mass fraction of $0.2 \%$.

Since V338Ser remained a unique object in spite of a decade of searches for V361 Hya pulsators, we have recently been paying particular attention to candidates in this lowgravity region. Here we present the discovery of pulsations in J20136+0928 (Figure 1), which has an even lower surface gravity than V338 Ser, and the lowest of any sdB pulsator found to date.

\section{TARGET SELECTION}

Since 2008 we have observed UV-excess targets selected based on UV photometry from the Galaxy Evolution Explorer (GALEX) satellite (Martin et al. 2005). An estimate based on targets with NUV $<14.0$ and FUV $-\mathrm{B}<+0.5$ is that only about two-thirds were already cataloged. UV-excess objects in this cut include a host of interesting compact stars. All types of pulsating WDs and hot subdwarf stars are included, as are most types of cataclysmic variables and planetary nebula nuclei. Our first cut based on GALEX data release 4 (GR4), contained 649 objects of which only about 400 had even approximate classes in the literature. We started observing targets from this list as a backup programme for various observing runs, and have by now mostly completed the sample. Full details of this survey will be given in a forthcoming paper. A similar survey is described by Vennes et al. (2011). As we have accumulated spectroscopic observations, new sdBV candidates have entered our observing lists for photometric follow-up, and new discoveries are emerging. The first result was the discovery of pulsations in J08069+1527, recently presented by Baran et al. (2011), and establishing it as a being a high-amplitude member of the rare DW Lyn (hybrid) type of sdBVs.

The object presented here appears in GALEX data release 6 (GR6) as GALEX J201337.6+092801, with UV magnitudes $\mathrm{FUV}=12.263(3), \mathrm{NUV}=13.079(3)$. Photographic magnitudes for this star from the NOMAD database are $B=12.01$, $V=12.22, R=12.34$, and from the 2MASS near-IR survey; $J=13.00, H=13.11, K=13.23$. The magnitudes are all perfectly consistent with an sdB star that has no main sequence companion. J20136+0928 also appears in the Tycho- 2 catalog as TYC 1077-218-1, where it has $B=12.16, V=11.78$. The NOMAD database also lists the target with a small but significant proper motion $\left(\mu_{\alpha}, \mu_{\delta}=12.1,2.8 \mathrm{mas} / \mathrm{yr}\right)$.

The target was observed spectroscopically during a run at the Isaac Newton Telescope (INT) at Observatorio Roque de los Muchachos on the island of La Palma (Spain). After the observing run we processed all the data and extracted the spectra with IRAF in the regular way. We fitted the hot subdwarfs using the pure H+He LTE model grids of Heber et al. (2000), and we show the resulting fit for J20136+0928 in Figure 2 The formal fitting errors (shown on the figure) do not account for systematic effects inherent in the models, so we generously increase the errors by a factor five when stating $T_{\text {eff }}=32100 \pm 1000, \log g=5.15 \pm 0.20$, and $\log \left(N_{\mathrm{He}} / N_{\mathrm{H}}\right)=-$ $2.8 \pm 0.2$. Note that the typical shift of when going from LTE to NLTE models is $\sim+1000 \mathrm{~K}$, and can become larger when metalicity effects are taken into account (see Heber 2009, and references therein). However, the low surface gravity of this object is clear.

\section{PHOTOMETRIC OBSERVATIONS}

The discovery run was made on October 6, 2010 with the 1.2-m Mercator Telescope, sited just next to the INT. Al- 


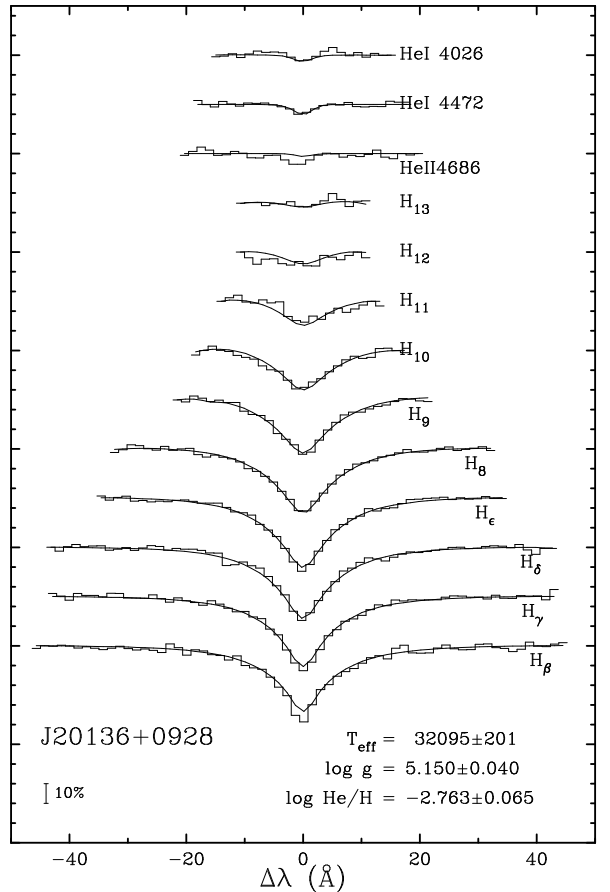

FIG. 2. — LTE model fit of J20136+0928.

though the conditions were poor, the main period of $\sim 520 \mathrm{~s}$ was clearly seen, and recognised to be longer than normal for a V361 Hya star. Since it was already clear from the spectroscopy that the gravity of the target was unusually low, the connection with V338 Ser was immediately made, and we urgently proceeded to confirm the discovery. As the target was already setting early in the night, we pooled observing time at several telescopes in order to collect enough photometry for a frequency analysis.

Photometric data were collected at three different sites within one month of the discovery run. At the Mercator telescope we used the Merope CCD camera, which was recently upgraded with a new E2V frame transfer CCD with $2048 \times 3074$ illuminated pixels (Østensen 2010). The observations were done with a Geneva- $B$ filter $(\lambda=4201 \pm 286)$, and a cadence period of $\sim 15 \mathrm{sec}$. With the $0.4-\mathrm{m}$ telescope at Baker Observatory (Missouri, USA) we used a Photometrics RS-1340 CCD with a BG40 filter $(\lambda=4750 \pm 1500)$, and a cadence of $\sim 30 \mathrm{~s}$. We also obtained three consecutive nights with the 0.84-m telescope at San Pedro Mártir (SPM) observatory in Mexico. The CCD camera is equipped with an E2V $4240 \mathrm{CCD}$, and we used a Bessel- $B$ filter $(\lambda=4350 \pm 980)$, and a cadence of $38 \mathrm{~s}$.

The light curves are all shown in Figure1, with the discovery run on top. Poor weather at Mercator left us unable to collect a significant amount of data at our prime site. Differential photometry was made with respect to the sum of three or four of the brightest reference stars found within the field of view of the CCDs. Most of this reference signal comes from TYC 1077-402-1, about 1.7' SW of the target, which is comparable in brightness to J20136+0928. The five bottom panels are consecutive nights, but the coverage is rather poor due to the target only being visible for a few hours before setting. On both October 27 and 28, there was a few hours of overlap between data from Baker and SPM. This allowed us to confirm that there is no significant difference in amplitude between observations made with the two telescopes, in spite

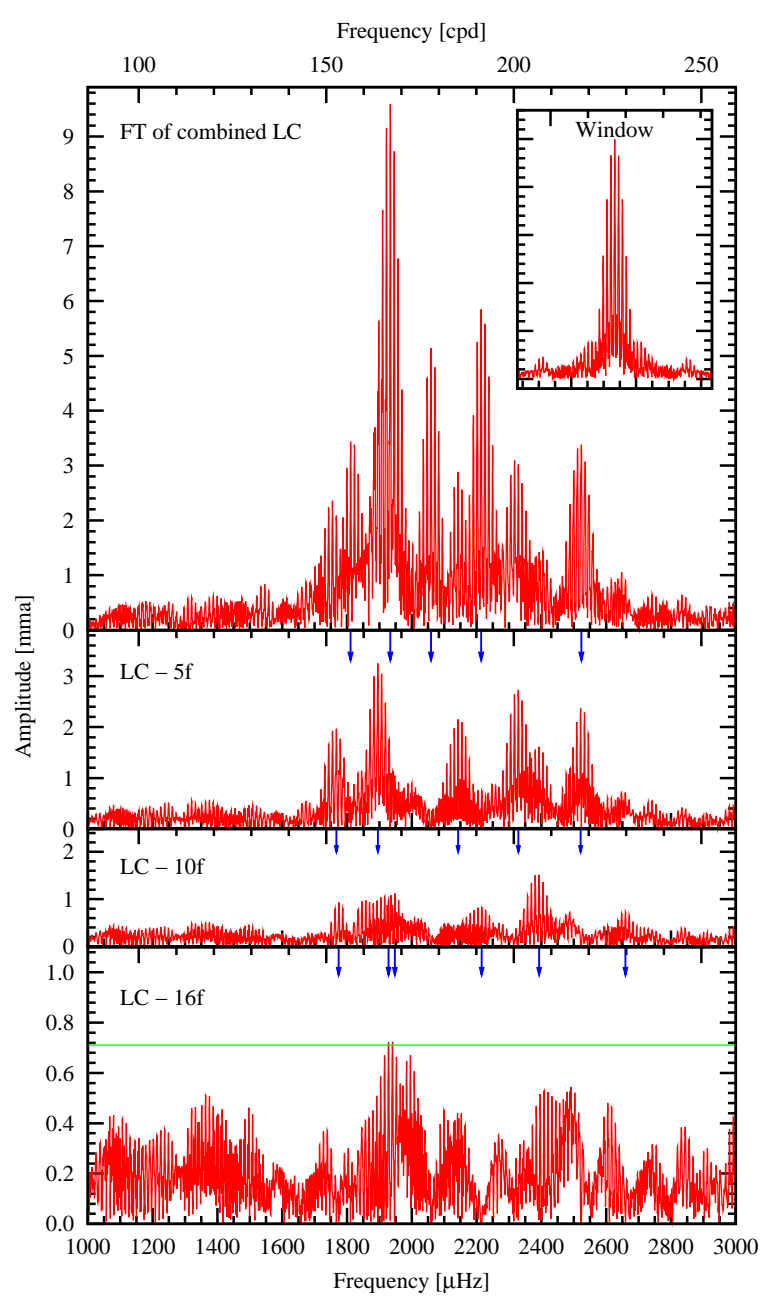

FIG. 3.- FT of the five consecutive nights taken together (top panel), and the prewhitening sequence after fitting and subtracting five, ten and sixteen frequencies from the original light curve. The window function is shown as an inset to the top panel. The arrows indicate the positions of the frequencies identified and removed in each step. The scale in the bottom panel has been expanded to show the residual level, and the line at which we stop is at 3.7 times the mean residual level.

of a significantly broader bandpass at Baker. We only use the last five nights for the frequency analysis described in the next section.

\section{FREQUENCY ANALYSIS}

The Fourier transform (FT) of all runs longer than $1 \mathrm{~h}$ is far too short to resolve the complexity of the frequency spectrum displayed by J20136+0928. Joining the five consecutive nights and taking the FT of the combined dataset produces the complex frequency spectrum with strong 1-day aliasing shown in the top panel of Figure 3. The tools used for the frequency determination were developed particularly for multisite data, and are described in Vučković et al. (2011). We proceed to prewhiten the most significant well-separated peaks, five or six at a time, until we reach a level of $0.71 \mathrm{mma}$ (straight line in the bottom panel of the same figure). This level is 4.2 times the noise level when computed in the highfrequency region where no pulsational power is seen (although we do detect the drive frequency of some of our telescopes), and 3.7 times the mean residual level of the 1000 to $3000 \mu \mathrm{Hz}$ region shown in the last panel. In each step the most significant peaks are used as input for a non-linear least- 


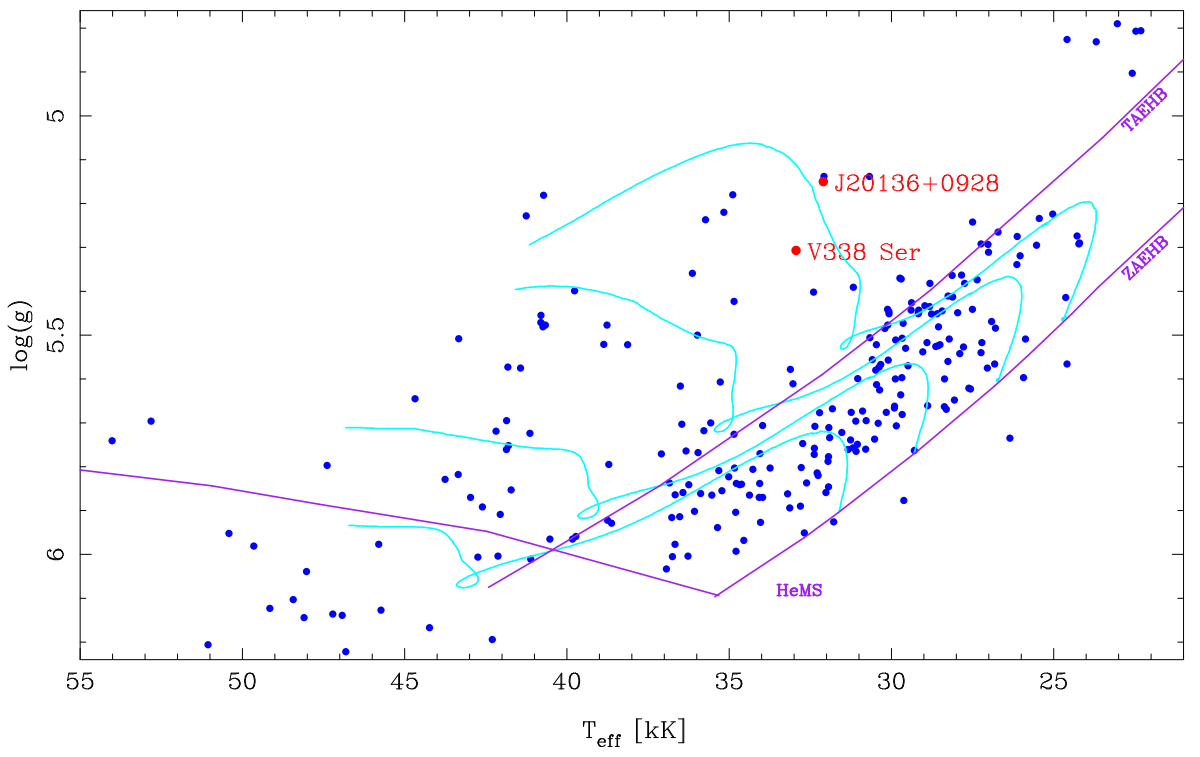

FIG. 4.- The $T_{\text {eff }}-\log g$ diagram as observed by the Bok-Green survey (Green et al. 2008) with V338 Ser highlighted, and J20136+0928 added. The evolutionary tracks are from Kawaler \& Hostler (2005).

TABLE 1

LIST OF FREQUENCIES

\begin{tabular}{|c|c|c|c|c|}
\hline ID & $\begin{array}{c}\text { Frequency } \\
(\mu \mathrm{Hz})\end{array}$ & $\begin{array}{l}\text { Period } \\
\text { (s) }\end{array}$ & $\begin{array}{c}\text { Amplitude } \\
\text { (mma) }\end{array}$ & $\begin{array}{l}\text { Phase } \\
\text { (s) }\end{array}$ \\
\hline$\overline{f_{9}}$ & $1767.3 \pm 0.3$ & $565.84 \pm 0.09$ & $2.18 \pm 0.38$ & $194 \pm 27$ \\
\hline$u_{15}$ & $1774.5 \pm 0.6$ & $563.53 \pm 0.19$ & $1.28 \pm 0.43$ & $12 \pm 56$ \\
\hline$f_{4}$ & $1810.7 \pm 0.2$ & $552.26 \pm 0.06$ & $3.91 \pm 0.42$ & $123 \pm 18$ \\
\hline$f_{7}$ & $1894.8 \pm 0.4$ & $527.75 \pm 0.11$ & $3.07 \pm 0.87$ & $246 \pm 40$ \\
\hline$u_{12}$ & $1908.1 \pm 0.7$ & $524.08 \pm 0.18$ & $1.84 \pm 0.71$ & $344 \pm 88$ \\
\hline$f_{1}$ & $1933.8 \pm 0.1$ & $517.12 \pm 0.04$ & $8.51 \pm 0.82$ & $139 \pm 19$ \\
\hline$u_{11}$ & $1946.9 \pm 0.5$ & $513.65 \pm 0.14$ & $2.11 \pm 1.03$ & $466 \pm 54$ \\
\hline$f_{3}$ & $2059.3 \pm 0.1$ & $485.60 \pm 0.03$ & $4.59 \pm 0.36$ & $82 \pm 11$ \\
\hline$f_{10}$ & $2142.8 \pm 0.3$ & $466.67 \pm 0.06$ & $2.11 \pm 0.36$ & $97 \pm 22$ \\
\hline$f_{2}$ & $2214.1 \pm 0.3$ & $451.66 \pm 0.05$ & $5.44 \pm 1.15$ & $70 \pm 29$ \\
\hline$u_{14}$ & $2215.2 \pm 1.0$ & $451.42 \pm 0.19$ & $1.45 \pm 1.18$ & $31 \pm 107$ \\
\hline$f_{8}$ & $2328.8 \pm 0.2$ & $429.41 \pm 0.04$ & $2.88 \pm 0.35$ & $103 \pm 15$ \\
\hline$f_{13}$ & $2392.9 \pm 0.4$ & $417.91 \pm 0.07$ & $1.51 \pm 0.35$ & $405 \pm 28$ \\
\hline$f_{6}$ & $2521.7 \pm 0.4$ & $396.56 \pm 0.06$ & $3.16 \pm 0.99$ & $31 \pm 37$ \\
\hline$f_{5}$ & $2523.3 \pm 0.3$ & $396.30 \pm 0.05$ & $3.86 \pm 0.99$ & $0 \pm 30$ \\
\hline$u_{16}$ & $2659.5 \pm 0.7$ & $376.02 \pm 0.10$ & $0.75 \pm 0.35$ & $26 \pm 50$ \\
\hline
\end{tabular}

squares fitting procedure, and all 16 frequencies, amplitudes and phases are left free in the final step. Several frequencies close to the most significant peaks do not converge to reliable values, and we consider these as uncertain (IDs labeled $u$ in Table 11. It is not clear whether this is due to the poor coverage of our data, or caused by real amplitude variability of the target. Concern can also be raised about some frequencies that are barely resolved (e.g. $f_{5}$ and $f_{6}$ ).

\section{DISCUSSION AND CONCLUSIONS}

A new, bright sdBV with an exceptionally low gravity has been found in a sample of UV-excess stars identified from GALEX photometry. In Figure 4 we show its position with respect to V338 Ser and the bulk of the sdB population, which falls between the zero and terminal age EHB lines. J20136+0928 is located close to minimum $\log g$ during the post-EHB stage of the uppermost evolutionary track, which corresponds to a $0.470 \mathrm{M}_{\odot}$ helium burning star with an $\mathrm{H}$-envelope mass of $0.004 \mathrm{M}_{\odot}$. Evolutionary models of more massive EHB stars are not shown, but can be found in Han et al. (2002), from which one may infer that models with a mass of $\sim 0.7 \mathrm{M}_{\odot}$ and an envelope of $\sim 0.01 \mathrm{M}_{\star}$ would approximate the position of $\mathbf{J} 20136+0928$ reasonably well. As mentioned in the introduction, such an overmassive EHB star cannot form through the regular binary mass-transfer scenarios that are responsible for producing the bulk of the EHB stars, but can form through the merger of two helium core WDs. Theoretical models for these scenarios, combined with future observational efforts, may allow us to determine the origins of both V338 Ser and J20136+0928.

This discovery brings the total number of short period sdBV stars (both V361 Hya and hybrid DW Lyn stars) up to 52 (49 are summarised in Østensen et al. 2010b, one was recently discovered in the field of the Kepler spacecraft by Kawaler et al. 2010, and the last one by Baran et al. 2011). The discovery of pulsations in a star with such a low gravity as $\mathbf{J} 20136+0928$ is well explained by the classical $\kappa$ mechanism for pulsations in sdBVs (Charpinet et al. 2001). However, the exceptionally low gravity of J20136+0928 is surprising in evolutionary terms, and the presence of pulsations allows the mass and internal structure to be explored. The encouraging brightness of J20136+0928 makes it an excellent target for time-resolved spectroscopy studies, and arranging a multi-site campaign with small telescopes when the target is visible throughout the night will be a priority for the coming season. By accurately determining the frequencies present in the star, asteroseismology will be able to reliably determine its mass and internal structure, and allow us to determine the most likely evolutionary origins of this object.

The research leading to these results has received funding from the European Research Council under the European Community's Seventh Framework Programme (FP7/20072013)/ERC grant agreement № 227224 (PROSPERITY), as well as from the Research Council of K.U.Leuven grant agreement GOA/2008/04. RO acknowledges financial support from the Spanish grants AYA2009-08481-E, AYA201014840 and AYA2009-14648-C02-02. AB gratefully appreciates funding from Polish Ministry of Science and Higher Ed- 
ucation under project № 554/MOB/2009/0. ACQ, JTG and LLH were supported by the Missouri Space Grant, funded by NASA. LFM acknowledges financial support from PAPIIT IN114309.
Based on observations made with the Mercator Telescope, operated on the island of La Palma by the Flemish Community, at the Spanish Observatorio del Roque de los Muchachos of the Instituto de Astrofísica de Canarias.

\section{REFERENCES}

Baran, A. S., Gilker, J. T., Reed, M. D., Østensen, R. H., Telting, J. H., Smolders K., Hicks L. \& Oreiro R. 2011, MNRAS, in press, doi:10.1111/j.1365-2966.2011.18356.x, arXiv:1103.1600

Charpinet, C., Fontaine, G., \& Brassard, P. 2001, PASP, 113, 775

Dorman, B., Rood, R. T. \& O’Connell, R. W. 1993, ApJ, 419, 596

For, B. Q., et al. 2010, ApJ, 708, 253, arXiv:0911.2006

Green, E. M., Fontaine, G., Hyde, E. A., For, B. Q. \& Chayer, P. 2008 in ASP Conf. Ser. 392, Hot Subdwarf Stars and Related Objects, ed. U Heber, C. S Jeffery \& R Napiwotzki (San Francisco, CA: ASP), 75

Han, Z., Podsiadlowski, P., Maxted, P. F. L. \& Marsh, T. R. 2003, MNRAS, 341, 669, arXiv:astro-ph/0301380

Han, Z., Podsiadlowski, P., Maxted., P. F. L., Marsh, T. R. \& Ivanova, N. 2002, MNRAS, 336, 449, arXiv:astro-ph/0206130

Heber, U. 1986, A\&A, 155, 33

Heber, U. 2009, ARA\&A, 47, 211

Heber, U., Reid, I. N. \& Werner, K. 2000, A\&A, 363, 198

Kawaler, S. D. \& Hostler, S. R. 2005, ApJ, 621, 432

Kawaler, S D, et al. 2010, MNRAS, 409, 1487, arXiv:1008.2356

Kilkenny, D, et al. 1999, MNRAS, 303, 525

Koen, C., O'Donoghue, D., Kilkenny, D., Lynas-Gray, A. E., Marang, F. \& van Wyk F. 1998, MNRAS, 296, 317
Martin, D. C., et al. 2005 ApJ, 619, L1

Maxted, P. F. L., Heber, U., Marsh, T. R. \& North, R. C. 2001 MNRAS, 326, 1391, arXiv:astro-ph/0103342

Napiwotzki, R., et al. 2004 in ASP Conf. Ser. 318, Spectroscopically and Spatially Resolving the Components of the Close Binary Stars ed. R. W Hilditch, H Hensberge \& K Pavlovski (San Francisco, CA: ASP), 402 Østensen, R. H. 2009, Communications in Asteroseismology, 159, 75, arXiv:0901.1618

Østensen, R. H. 2010, Astron. Nachr., 331, 1026, arXiv:1010.3214

Østensen, R. H., et al. 2010a, MNRAS, 408, L51, arXiv:1006.4267

Østensen, R. H., et al. 2010b, A\&A, 513, A6, arXiv:1001.3657

Østensen, R. H., Telting, J. H., Oreiro, R., Heber, U., de Beck, E. \& Reed, M. 2010c, Ap\&SS, 329, 167

Reed, M. D. \& Stiening, R. 2004, PASP, 116, 506

Tillich, A., Heber, U., O’Toole, S. J., Østensen, R. \& Schuh, S. 2007, A\&A, 473, 219, arXiv:0707.0810

van Grootel, V., Charpinet, S., Fontaine, G. \& Brassard, P. 2010, Ap\&SS, 329,217

Vennes, S., Kawka, A. \& Németh, P. 2011, MNRAS, 410, 2095, arXiv: 1008.3823

Vučković, M., et al. 2006, ApJ, 646, 1230, arXiv:astro-ph/0604330 